\title{
Auf dem Weg zu einer Sorgeökonomie
}

\author{
Ulrike Knobloch
}

Unter Ökonomie wird meist die Erwerbswirtschaft, die bezahlte Ökonomie verstanden. Von verschiedenen Seiten wird dagegen eine Erweiterung um die Versorgungswirtschaft, die unbezahlte Ökonomie nicht nur gefordert, sondern auch vorgenommen. Die dabei entstehenden Ansätze lassen sich unter dem Begriff "Sorgeökonomie « zusammenfassen. Ihr Kennzeichen ist, dass sie Erwerbs- und Versorgungswirtschaft, bezahlte und unbezahlte Ökonomie, verbinden. Die Sorgeökonomie ist danach kein Spezialgebiet der Ökonomie, sondern liefert einen Ansatz zu einer allgemeinen Wirtschaftstheorie.

Die Frage »Was ist Ökonomie? « scheint schnell beantwortet: Es geht um den effizienten Umgang mit knappen Mitteln, wobei in aller Regel nur die Erwerbswirtschaft interessiert, also der bezahlte und im Bruttoinlandsprodukt sichtbare Teil der Wirtschaft. Die vielfältigen, unbezahlt geleisteten Tätigkeiten bleiben nicht nur im Bruttoinlandsprodukt, sondern auch im Alltag häufig unsichtbar. (1) Doch auch in diesem unbezahlten Bereich wird ökonomisch gehandelt, insofern hier Lebenszeit sowie materielle und finanzielle Ressourcen direkt oder indirekt zur eigenen oder der Versorgung anderer Menschen eingesetzt werden.

Den unbezahlt geleisteten Teil der Wirtschaft nenne ich »Versorgungswirtschaft «, denn hier steht die Versorgung der Menschen mit dem zum (guten) Leben Notwendigen im Vordergrund, also der gesamte Bereich des Wirtschaftens im und um den Haushalt, insbesondere die Hausarbeit, die Betreuung und Pflege von Angehörigen, Nachbarschaftshilfe und viele andere Formen der Freiwilligenarbeit. Diese Tätigkeiten sind für jedes Wirtschaftssystem überlebenswichtig, so dass jede Gesellschaft ein großes Interesse daran haben muss, dass sie bereitgestellt werden (Jochimsen/Knobloch 1997).

Demnach ist es sinnvoll, den Ökonomiebegriff systematisch so zu erweitern, dass nicht nur die bezahlte Erwerbswirtschaft, sondern auch die unbezahlte Versorgungswirtschaft einbezogen ist. Denn der »Wohlstand der Nationen « (Adam Smith) wird nicht nur in der Erwerbswirtschaft, sondern in durchaus vergleichbarer Größenordnung auch in der Versorgungswirtschaft erzeugt (Statistisches Bundesamt 2004: 247 ff.).

Analog zum Ökonomiebegriff bezieht sich die weit verbreitete Vorstellung von Arbeit auf die bezahlten Tätigkei-

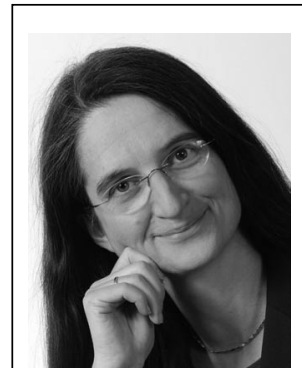

Dr. Ulrike Knobloch ist Oberassistentin am Departement Sozialarbeit und Sozialpolitik der Universität Freiburg in der Schweiz und Lehrbeauftragte an der Universität St. Gallen. Sie hat das »Netzwerk Vorsorgendes Wirtschaften « mitbegründet und ist Mitherausgeberin der Buchreihe »Lebensweltökonomie«. Ihre Forschungsschwerpunkte liegen in der Verbindung von Gender-Ökonomie und Ethik und umfassen Wirtschaftsethik und Sozialökonomie, Institutionelle Ökonomie und Ordnungspolitik, Sozialwirtschaft und Non-ProfitManagement sowie nachhaltiger Konsum.

E-Mail ulrike.knobloch@unifr.ch

ten in der Erwerbswirtschaft. Diese hier genauer als Erwerbsarbeit bezeichneten Tätigkeiten sind aber nicht die einzige Form von Arbeit. Mindestens ebenso bedeutsam ist die Versorgungsarbeit, die unbezahlt im und um den Haushalt geleistet wird. Versorgungsarbeit ist für den Einzelnen wie für die Gesellschaft notwendige Arbeit. Sie ist an anderen Zielen orientiert als die Erwerbsarbeit, denn es steht nicht die Einkommenserzielung, sondern die Versorgung der Menschen mit dem zum Leben Notwendigen im Vordergrund. Es handelt sich um Tätigkeiten, die aus der Einsicht heraus ausgeführt werden, dass sie für einen selbst oder für andere notwendig sind (vgl. Kasten« Wie man Arbeit definiert: das Drittpersonen-Kriterium«, Seite 16).

Durch empirische Untersuchungen, wie Zeitbudgeterhebungen, Satellitenkonten zur Haushaltsproduktion und Freiwilligensurveys, sind zum einen Umfang und Wert der unbezahlten Arbeit sichtbar gemacht worden, und zum anderen, damit verbunden, die immense Bedeutung, die diese Arbeit für jede Gesellschaft hat. Laut der deutschen Zeitbudgeterhebung von 2001/2002 beträgt das Jahresvolumen der unbezahlten Arbeit in Deutschland 96 Milliarden Stunden. Im Vergleich dazu hat die gesamte innerhalb des gleichen Jahres erbrachte bezahlte Arbeit mit 56 Milliarden Stunden ein deutlich geringeres Volumen. Auf eine Woche bezogen wird im Durchschnitt gut 25 Stunden unbezahlt und etwa 17 Stunden bezahlt gearbeitet, wobei das Wochenende mit eingerechnet wurde. Es wird also wesentlich mehr unbezahlt als bezahlt gearbeitet (Statistisches Bundesamt 2004). (2) 
Die Sorgeökonomie (engl.: care economy) versucht eine systematische Verbindung zwischen Erwerbswirtschaft und Versorgungswirtschaft herzustellen, wodurch die unbezahlte Arbeit, die auch heute noch überwiegend von Frauen geleistet wird, in ihrem Umfang und ihrer Bedeutung sichtbar wird. Es gibt allerdings nicht nur einen sorgeökonomischen Ansatz, sondern eine Vielzahl von Überlegungen, die ökonomische Theorie so zu erweitern, dass der gesamte unbezahlte Bereich mit seinen besonderen Merkmalen einbezogen ist. Diese Ansätze zur Sorgeökonomie unterscheiden sich durch einige Besonderheiten, Erweiterungen und Prämissen wesentlich von den traditionellen ökonomischen Ansätzen (Power 2004, Madörin 2006). Neben der Erweiterung des Ökonomieverständnisses sind dies insbesondere eine eigene methodische Herangehensweise und ein Handlungsmodell jenseits des eigeninteressierten Nutzenmaximierers (Knobloch 2006, 2008).

Maren Jochimsen hat eine ökonomische Sorgetheorie entworfen, um Sorgesituationen und ihre spezifischen Kennzeichen in der ökonomischen Wissenschaft angemessen erfassen zu können. Ausgangspunkt für ihre Theorie des Sorgens bildet die Bereitstellung von Sorgetätigkeiten insbesondere für Kinder, ältere, kranke und behinderte Menschen. Um die Besonderheit von Sorgesituationen deutlich hervorheben $\mathrm{zu}$ können, geht sie von extremen Sorgesituationen aus, die gekennzeichnet sind durch eingeschränkte oder nicht vorhandene Handlungsfähigkeit und damit begrenzte Autonomie sowie durch sich daraus ergebende Asymmetrien und Abhängigkeiten (Jochimsen 2003a, 2003b).

Für die Bereitstellung von Sorgetätigkeiten ist es wichtig, zwei Bestandteile dieser Tätigkeiten zu unterschieden: das instrumentelle Element, also die konkrete sorgende Tätigkeit, beispielsweise spezielle Handgriffe in der Pflege, und das kommunikative Element, also die ergänzende, aber ebenso wichtige Tätigkeit, die die instrumentelle Sorgetätigkeit begleitet, beispielsweise das Sprechen mit der umsorgten Person. Erst beide Elemente zusammen, die in der Fachliteratur als integratives Produkt bezeichnet werden (Folbre 1995), sichern die Qualität der Sorgetätigkeit (Jochimsen 2003b: 45).

\section{Sozialwirtschaft zwischen Erwerbs- und Versorgungswirtschaft}

Indem die Theorie des Sorgens dem Konzept der Asymmetrie zentrale analytische Bedeutung beimisst, werden die in der Standardökonomie dominierenden symmetrischen Tauschverhältnisse zu einem Sonderfall von Asymmetrie (Jochimsen 2003b: 39). Mit der Umkehrung der Prämissen - Autonomie und Unabhängigkeit als Spezialfall, beschränkte Handlungsfähigkeit und Abhängigkeiten als Normalfall - wird ein neues Handlungsmodell, das für eine Sorgeökonomie und damit auch für eine neue allgemeine ökonomische Theorie einen wesentlichen Ansatzpunkt liefert, umrissen.

Welche Rolle kommt nun der Sozialwirtschaft bei der Entwicklung einer Sorgeökonomie als allgemeiner Wirt- schaftstheorie zu? Welche Position nimmt die Sozialwirtschaft zwischen Erwerbs- und Versorgungswirtschaft ein?

In dem Umfang, wie sie bezahlte Arbeitsplätze zur Verfügung stellt, ist die Sozialwirtschaft Teil der Erwerbswirtschaft. Gleichzeitig bleibt sie auch in ihren bezahlten Bereichen den Zielen und Inhalten der Versorgungswirtschaft nahe. Zudem wird in der Sozialwirtschaft viel Freiwilligenarbeit geleistet, die einen Teil der Versorgungswirtschaft bildet. Zwischen bezahlter Erwerbswirtschaft und unbezahlter Versorgungswirtschaft hat die Sozialwirtschaft also eine Zwitterposition inne: Sie ist Teil der bezahlten Erwerbswirtschaft, denn sie stellt in großem Maße Arbeitsplätze zur Verfügung (Zimmer/Nährlich 2003: 77f., IW 2004). Und sie ist ein Teil der Versorgungswirtschaft, denn sie greift in großem Umfang auf institutionalisierte, aber auch informelle Freiwilligenarbeit zurück (siehe Abbildung Seite 16.).

Doch auch in ihrem erwerbswirtschaftlichen Teil steht die Sozialwirtschaft der Versorgungswirtschaft nahe. Denn auch in ihrem erwerbswirtschaftlichen Teil stellt sie die Versorgung der Menschen mit dem zum Leben Notwendigen in den Vordergrund. Die Schreibweise des Titels 
Tätigkeiten in die unbezahlte Versorgungswirtschaft ausgelagert (Entmarktlichungsprozesse).

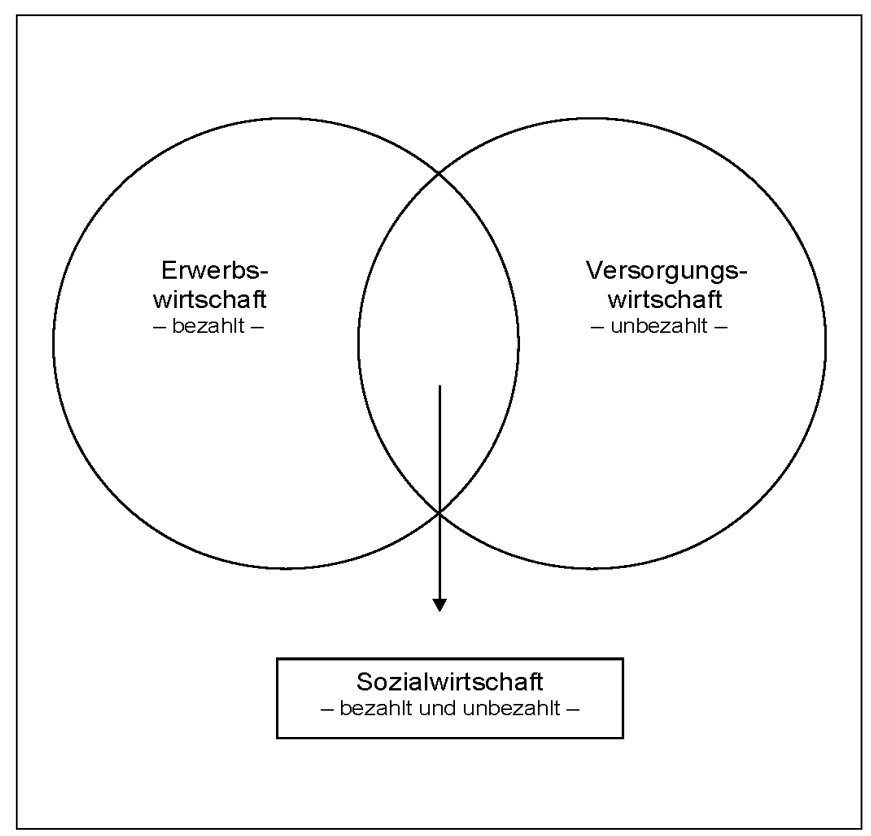

\section{Wie man Arbeit definiert:} das Drittpersonen-Kriterium

Um nicht zahlreiche im Wirtschaftsleben notwendige Leistungen unberücksichtigt zu lassen, tritt an die Stelle eines rein an der Erwerbswirtschaft orientierten Arbeitsbegriffs ein erweiterter Arbeitsbegriff, der unter Einbezug aller gesellschaftlich notwendigen Arbeiten erwerbs- und versorgungswirtschaftliche Tätigkeiten umfasst. Arbeit wird dann von anderen Tätigkeiten nicht mehr über das Einkommen abgegrenzt, sondern mit Hilfe des Drittpersonen-Kriteriums:

Ob etwas Arbeit ist oder nicht, entscheidet sich danach, ob es sich um Tätigkeiten handelt, die auch von einer dritten Person übernommen werden können - dann handelt es sich um Arbeit - oder ob man sie selbst tun muss, um in den Genuss der Tätigkeit zu kommen - dann handelt es sich nicht um Arbeit.

Nach dem Drittpersonen-Kriterium sind somit alle Aktivitäten Arbeit, die an Dritte delegiert werden können. Beispielsweise sind Mahlzeiten zubereiten und Wäsche waschen, denn diese Tätigkeiten können auch von anderen ausgeführt werden, Essen und Schlafen sind dagegen keine Arbeit, denn das muss jede und jeder selbst tun, um satt und ausgeschlafen sein.

\section{Ulrike Knobloch}

\section{Vermarktlichungsprozesse}

Mithilfe des Drittpersonen-Kriteriums haben wir die Tätigkeiten bestimmt, die als Arbeit bezeichnet werden und dadurch gekennzeichnet sind, dass sie grundsätzlich auch von einer anderen Person ausgeführt werden könnten. Es sind insbesondere Kinderbetreuung, Pflege von Angehörigen, Mahlzeitenzubereitung, Waschen und Bügeln. Eine Vermarktlichung findet statt, wenn diese Güter und Dienstleistungen, die bisher von Haushaltsmitgliedern oder der Zivilgesellschaft produziert wurden, von Einzelpersonen oder erwerbswirtschaftlichen Einrichtungen gegen Bezahlung übernommen werden.

Viele der früher unbezahlt verrichteten Tätigkeiten werden heute nicht mehr von den Haushaltsmitgliedern oder der Zivilgesellschaft erbracht, sondern von Personen, die diese Tätigkeiten gegen - meist geringe - Bezahlung im Haushalt leisten. Zu den haushaltsexternen Personen, die diese Dienstleistungen im Haushalt übernehmen, gehören auch immer mehr Frauen (seltener Männer) aus anderen Kulturkreisen. Deren Anteil an versorgungswirtschaftlichen Tätigkeiten im Heimatland wird dann vonmeist wiederum weiblichen - Verwandten, Nachbarn oder Freunden erbracht. (3) Bei dieser Form der Verschiebung bleibt der Ort der Leistungserstellung der private Haushalt, der dadurch in zunehmendem Maße zum Erwerbsarbeitsplatz wird (Gather/Geissler/Rerrich 2002).

Eine andere Form der Verschiebung von unbezahlter Versorgungsarbeit in die Erwerbswirtschaft besteht darin, versorgungswirtschaftliche Tätigkeiten aus dem Haushalt oder anderen versorgungswirtschaftlichen Bereichen in die Erwerbswirtschaft zu verlagern. Auf lokaler Ebene sind dies beispielsweise Essens- und Pflegedienst, Kinderhort, Wäscherei, Partyservice. Auf globaler Ebene werden versorgungswirtschaftliche Tätigkeiten zunehmend durch die Produktpalette weltweit agierender Unternehmen etwa der Pflege- und Lebensmittelbranche, deren Angebot von Halbfertigprodukten bis hin zu Pflegerobotern reicht, oder auch durch Fastfood-Ketten ergänzt oder ersetzt.

Gemeinsam ist beiden Formen des Vermarktlichungsprozesses, dass bisher unbezahlt geleistete Tätigkeiten jetzt bezahlt in der Erwerbswirtschaft geleistet werden. Dass die Versorgungswirtschaft auf diese Weise vermarktlicht wird, ist historisch nicht neu. Doch durch zwei Entwicklungen erhält diese Richtung des Verlagerungsprozesses eine ganz neue Dimension, zum einen durch die immense wirtschaftliche Globalisierung und zum anderen durch die zunehmende Erwerbsbeteiligung von Frauen, ohne dass Männer im gleichen Umfang in der Versorgungswirtschaft tätig werden.

\section{Entmarktlichungsprozesse}

In umgekehrter Richtung wirken die Auslagerungsprozesse erwerbswirtschaftlicher Tätigkeiten in die unbezahlte Versorgungswirtschaft. Beschleunigt werden diese Verschiebungen durch die Verbreitung der modernen Informationsund Kommunikationstechnologien. Dadurch können eine Vielzahl von Tätigkeiten, die früher in der Erwerbswirtschaft erbracht wurden, jetzt von den privaten Haushalten 
übernommen werden, beispielsweise durch Online-Banking, durch virtuelle medizinische Voruntersuchungen, durch Reisebuchungen per Internet $\mathrm{u}$. v. m. Den Vorteilen der Kostenersparnis und der Zeitunabhängigkeit auf der einen Seite steht dabei der zusätzliche finanzielle und zeitliche Aufwand auf der anderen Seite gegenüber.

Eine andere Form der Entmarktlichungsprozesse sind die Leistungsverschiebungen, die aufgrund knapper öffentlicher Mittel erfolgen, wenn beispielsweise Patienten so früh aus dem Krankenhaus entlassen werden, dass sie zu Hause auf weitere Pflege angewiesen sind, oder Kinder wieder zu Hause betreut werden, weil Kinderkrippen schließen mussten.

Doch die Verlagerung von Tätigkeiten in die unbezahlte Versorgungswirtschaft stößt an Grenzen. Denn während die Berufstätigkeit von Frauen in den vergangenen Jahrzehnten immer weiter zugenommen hat, hat sich der Anteil, den Männer an den versorgungswirtschaftlichen Tätigkeiten übernehmen, laut der Zeitbudgeterhebung 2001/2002 nicht wesentlich erhöht (Statistisches Bundesamt 2004: 251 ff.). Wenn die bisherige geschlechtsspezifische Arbeitsteilung bestehen bliebe, hätte die Verlagerung zusätzlicher erwerbswirtschaftlicher Tätigkeiten in die Versorgungswirtschaft daher unweigerlich zur Folge, dass entweder

- die Doppelbelastung und die damit einhergehende Zeitarmut der sowohl in Erwerbs- als auch in Versorgungsökonomie Beschäftigten weiter zunähme oder

- zusätzliche versorgungswirtschaftliche Tätigkeiten in die Erwerbswirtschaft ausgelagert werden müssten oder

- sich die Zeit für versorgungswirtschaftliche Tätigkeiten insgesamt (weiter) verringert und dadurch eine Versorgungslücke entstehen könnte.

\section{Fazit}

Die Sorgeökonomie stellt eine systematische Verbindung von erwerbswirtschaftlichen, über den Markt vermittelten Tätigkeiten und versorgungswirtschaftlichen, in und um den Haushalt koordinierten Tätigkeiten her und macht die Verschiebungen, die zwischen diesen beiden Bereichen stattfinden, sichtbar. Die Sozialwirtschaft, die im Schnittfeld von Erwerbs- und Versorgungswirtschaft steht, kann dabei eine zentrale Vermittlerrolle einnehmen, wenn sie diese Aufgabe in ihrer Relevanz für die ökonomische Theoriebildung begreift und Sorgeökonomie als allgemeine Wirtschaftstheorie versteht.

Dabei wird gerade durch die Sozialwirtschaft der Blick auch auf die Anbieterstruktur gelenkt, denn soziale Dienstleistungen werden ja nicht nur vom Staat, sondern auch von Unternehmen, Haushalten und dem Dritten Sektor erbracht. Gerade vor dem Hintergrund, dass Frauen möglichst gleichberechtigt im Erwerbsarbeitsmarkt teilnehmen sollen und Männer sich nicht in einem vergleichbaren Umfang an der entsprechenden Versorgungsarbeit, also Pflege und Betreuung von Angehörigen zu Hause beteiligen (können), erscheint mir die Frage, wer in Zukunft welche sozialen Aufgaben übernehmen soll, mehr als zentral.

\section{Anmerkungen}

(1) Insbesondere von der feministischen Ökonomie wird eine Erweiterung des Ökonomiebegriffs um die Versorgungswirtschaft, die unbezahlte Ökonomie nicht nur gefordert, sondern auch vorgenommen - wobei ich lieber von genderbewusster Ökonomie spreche, um dem Missverständnis, dass es sich dabei um einen Teilbereich handeln würde, zu entgehen; siehe Busch-Lüty u. a. (1994), Himmelweit (1995), Folbre (1995), Biesecker u.a. (2000), Jochimsen (2003a,b), Jochimsen u.a. (2004), Jochimsen/Knobloch (2006), Madörin (2006), Razavi (2007).

(2) Die Zeitbudgeterhebung von 2001/2002 hat zudem ergeben, dass Frauen im Durchschnitt pro Woche 12 Stunden und Männer 22,5 Stunden bezahlt arbeiten, dass Frauen pro Woche 31 Stunden und Männer 19,5 Stunden unbezahlt arbeiten, dass Frauen insgesamt, also unbezahlte und bezahlte Arbeit zusammen genommen, mit 43 Stunden eine Stunde mehr arbeiten als Männer mit 42 Stunden (Statistisches Bundesamt 2004).

(3) In der Einleitung zu ihrem Buch »Weltmarkt Privathaushalt « schreiben Claudia Gather, Birgit Geissler und Maria Rerrich dazu: »Der Privathaushalt ist heute ein Weltmarkt für weibliche Arbeitskräfte: die Putzfrauen aus Polen und Kroatien, die Kinderfrauen aus Brasilien und Ecuador, die Aupairs aus der Ukraine und Ungarn. Aber auch viele v. a. ältere deutsche Frauen sind hier beschäftigt; nach langjähriger Tätigkeit in der einen Rolle als >dienstbare Geister`, als Hausfrauen, wechseln sie in die andere Rolle als >dienstbare Geister`, als Putzfrauen.« (Gather/Geissler/ Rerrich 2002: 8).

\section{Literatur}

Biesecker, Adelheid/Mathes, Maite/Schön, Susanne/Scurrell, Babette (Hg.) (2000): Vorsorgendes Wirtschaften. Auf dem Weg zu einer Ökonomie des Guten Lebens, Bielefeld: Kleine Verlag.

Busch-Lüty, Christiane/Jochimsen, Maren/Knobloch, Ulrike/Seidl, Irmi (Hg.) (1994): Vorsorgendes Wirtschaften. Frauen auf dem Weg zu einer Ökonomie der Nachhaltigkeit, Politische Ökologie, Sonderheft 6, München: ökom.

Folbre, Nancy (1995): Holding Hands at Midnight. The Paradox of Caring Labor, in: Feminist Economics 1 (1), 73-92.

Gather, Claudia/Geissler, Birgit/Rerrich, Maria S. (Hg.) (2002): Weltmarkt Privathaushalt. Bezahlte Haushaltsarbeit im globalen Wandel, Münster: Westfälisches Dampfboot.

Himmelweit, Susan (1995): The Discovery of $>$ Unpaid Work . The Social Consequences of the Expansion of >Workı, in: Feminist Economics 2, No. 2, 1-19. 
IW - Institut der deutschen Wirtschaft (2004): Auf den Schultern der Schwachen. Wohlfahrtsverbände in Deutschland, Köln.

Jochimsen, Maren A. (2003a): Careful Economics. Integrating Caring Activities and Economic Science, Boston, Dordrecht, London: Kluwer.

Jochimsen, Maren A. (2003b): Die Gestaltungskraft des Asymmetrischen. Kennzeichen klassischer Sorgesituationen und ihre theoretische Erfassung in der Ökonomik, in: Zeitschrift für Wirtschafts- und Unternehmensethik 4, Heft 1: Themenschwerpunkt: Feministische Wirtschaftsethik, 38-51.

Jochimsen, Maren A./Kesting, Stefan/Knobloch, Ulrike (Hg.) (2004): Lebensweltökonomie, Bielefeld: Kleine Verlag.

Jochimsen, Maren A./Knobloch, Ulrike (2006): Lebensweltökonomie in Zeiten wirtschaftlicher Globalisierung, Bielefeld: Kleine Verlag.

Jochimsen, Maren A./Knobloch, Ulrike (1997): Making the Hidden Visible: The Importance of Caring Activities and their Principles for any Economy, in: Ecological Economics, Special Issue: Women, Ecology and Economics, Vol. 20/2, 1997, 107-112.

Knobloch, Ulrike (2008): Ansatzpunkte einer Sorgeökonomie als allgemeine Wirtschaftstheorie: Genderbewusste Wirtschaftsethik - Lebensweltökonomie Vorsorgendes Wirtschaften, in: Judith Dellheim/ Günther Krause (Hg.): Für eine neue Alternative. Herausforderungen einer sozialökologischen Transformation. Berlin: Dietz (im Erscheinen).
Knobloch, Ulrike (2006): Was ist Ökonomie? Genderbewusste Wirtschaftsethik - Vorsorgendes Wirtschaften - Lebensweltökonomie, in: Carola Möller/Ursula Peters/Irina Valley (Hg.): Dissidente Praktiken. Erfahrungen mit herrschafts- und warenkritischer Selbstorganisation, Königstein Ts.: Ulrike Heimer Verlag, 206-211.

Madörin, Mascha (2006): Plädoyer für eine eigenständige Theorie der Care-Ökonomie, in: Torsten Niechoj/Marco Tullney (Hg.): Geschlechterverhältnisse in der Ökonomie, Marburg: Metropolis, 277-297.

Power, Marilyn (2004): Social Provisioning as a Starting Point for Feminist Economics, in: Feminist Economics 10 (3), 3-19.

Razavi, Shahra (2007): The Political and Social Economy of Care in a Development Context. Conceptual Issues, Research Questions and Policy Options, Gender and Development Programme Paper Number 3, Genf: UNRISD.

Statistisches Bundesamt (2004): Alltag in Deutschland. Analysen zur Zeitverwendung. Beiträge zur Ergebniskonferenz der Zeitbudgeterhebung 2001/02 am 16./17.2.2004, Forum der Bundesstatistik Band 43, Wiesbaden.

Zimmer, Annette/Nährlich, Stefan (2003): Zur volkswirtschaftlichen Bedeutung der Sozialwirtschaft, in: Arnold, Ulli/Maelicke, Bernd (Hg.): Lehrbuch der Sozialwirtschaft, 2. Aufl., Baden-Baden: Nomos, S. 64-80.

\section{Wirksame Managementberatung, Projektrealisierung und Hilfsangebote für Ihren Erfolg!}

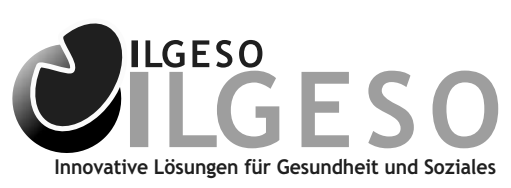

Wir beraten und coachen Ihre Führungskräfte, Verantwortlichen und Nachwuchsführungskräfte rund um das Thema Management, insbesondere in den Bereichen

- Wirksames Management, Führungskompetenz und Selbstmanagement

- Mitarbeiterführung und Motivation sowie Gesprächsführung und Humankommunikation.

Wir sind für Sie da, um für lhre Probleme und Herausforderungen individuelle und wirksame Lösungen zu erarbeiten, mit dem Ziel, Ihre Professionalität und Wirksamkeit für Ihren Erfolg zu erhöhen.

Zudem sind wir darauf spezialisiert, Ihre Ideen, Wünsche und Zielvorstellungen in realisierbare Implementierungskonzepte umzusetzen und diese wirksam und zeitnah in Ihre neuen innovativen Projekte und Angebote zu verwandeln, um Sie dabei zu unterstützen, Ihre Leistungs- und Angebotsvielfalt zu Ihrer nachhaltigen und erfolgreichen Weiterentwicklung zu erweitern.

Ergänzt wird unser Leistungsangebot durch Coaching-, Seminar- und Fortbildungsangebote rund um das Thema Management.

$$
\text { www.ilgeso.de info@ilgeso.de www.yocahe.de }
$$

